\title{
A model of multi-pass absorption of external EC radiation at initial stage of discharge in ITER
}

\author{
P.V. Minashin ${ }^{1, a}$, A.B. Kukushkin ${ }^{1,2}$, R.R. Khayrutdinov ${ }^{1,3}$, V.E. Lukash ${ }^{1}$ \\ ${ }^{1}$ Tokamak Physics Institute, NRC Kurchatov Institute, Moscow, Russia \\ ${ }^{2}$ National Research Nuclear University MEPhI, Moscow, Russia \\ ${ }^{3}$ Troitsk Institute for Innovation \& Fusion Research, Troitsk, Russia
}

\begin{abstract}
A model is developed for multi-pass absorption of external electron cyclotron radiation (ECR) in tokamaks, which is used at initial stage of discharge to overcome the impurity radiation barrier (burn-through). Model is based on a semi-analytical solution of the ECR transport problem in the case of multiple reflection of radiation from the wall of the vacuum chamber. We estimate the efficiency of absorption of injected radiation for typical values of the electron temperature and density at the initial stage of discharge in ITER.
\end{abstract}

\section{Introduction}

Due to technological issues the ohmic plasma breakdown in tokamak-reactor ITER is only possible over a narrow range of plasma pressure and magnetic field errors [1]. In this connection for the reliable plasma start-up in ITER it is planned to use the electron cyclotron resonance heating (ECH, ECH-assisted start-up) [2], [3]. The ECH is a standard way for plasma start-up in stellarators and already showed to be an effective tool for plasma breakdown in tokamaks [4].

The main functions of the ITER ECH\&CD system are as follows [3]:

- ECH-assisted plasma start-up: assistance to initial breakdown and the heating during the current ramp-up,

- auxiliary heating to achieve the $\mathrm{H}$ mode and the fusion energy gain factor $\mathrm{Q}=10$,

- steady state on-axis and off-axis current drive,

- MHD instabilities control by the localized current drive.

In ITER in a wide range of operating parameters all the requirements to the ECH\&CD-system may be fulfilled by using the fixed frequency gyrotrons $(170 \mathrm{GHz})$, launching elliptically polarized ordinary wave (O-mode) from the low magnetic field side. Basic parameters of the ITER EC-heating system are shown in fig. 1.

At the initial stage of the discharge due to the low electron temperature the absorption of the O-mode is very small. Therefore, to ensure the burn-through the ordinary wave is launched at an oblique angle to the magnetic field (for ITER, toroidal injection angle $\Phi \geq 20^{\circ}$ ), which leads to the conversion of ordinary mode to extraordinary (Xmode) one in the reflections of the EC radiation from the wall of the vacuum chamber [1]. The fundamental harmonic of the X-mode is strongly absorbed even at zero electron temperature, which makes it ideal for use in start-up scenarios [5]. It is assumed that after a few reflections of the O-mode from the wall (about 4 reflections) $75 \%$ of the injected power (if only the mode conversion take place) will be converted to X-mode and absorbed by the plasma [1].

There are alternative scenarios of RF heating at the initial stage of discharge. For example electron Bernstein waves (EBW) can be used for heating, because there is no density cut-off for this electrostatic waves and they are fully absorbed in the EC resonance zone. EBW can't exist without plasma, so EBW can't be launched from the antenna outside the plasma. One can use the X-mode to EBW conversion in the upper hybrid resonance (scheme $\mathrm{XB}$, namely, launch of the $\mathrm{X}$-waves from the high magnetic field side and its conversion to the Bernstein waves, and the OXB scheme, namely, launch of the Owave, its full conversion in the first wall reflection to the $\mathrm{X}$-wave and then conversion of the X-wave to EBW) [6].

Modelling of the initial stage of plasma discharge in ITER with the 0D model [1] showed that in a wide range of initial conditions, taking into account beryllium impurities, the $3 \mathrm{MW}$ of absorbed external EC radiation is needed to achieve the plasma breakdown (for the carbon impurities even $5 \mathrm{MW}$ of absorbed power is not enough). However, in [1] the efficiency of EC absorption was not calculated.

Modeling of ECH and ECCD was performed using kinetic codes (solution of the Fokker-Planck (FP) equation): OGRAY code [7]; or ray-tracing code GENRAY [8] + FP code CQL3D [9]. Also the simplified models were used [10], [11]. In all these calculations the single-pass absorption model has been used.

\footnotetext{
${ }^{\mathrm{a}}$ Corresponding author Minashin_PV$[$ at]nrcki.ru
} 
Recent 1D simulations [12] of the ECH start-up in ITER with the help of the OGRAY code [7] for the ECH calculations showed that the planned EC power could be not enough for plasma breakdown because of low efficiency of the single-pass ECH power absorption.

When the high power of ECH is injected at initial stage of discharge (low density plasma), we have to allow for multi-pass absorption. Here we propose a model for calculating the efficiency of the absorption of external EC power in tokamaks at initial stage of discharge.

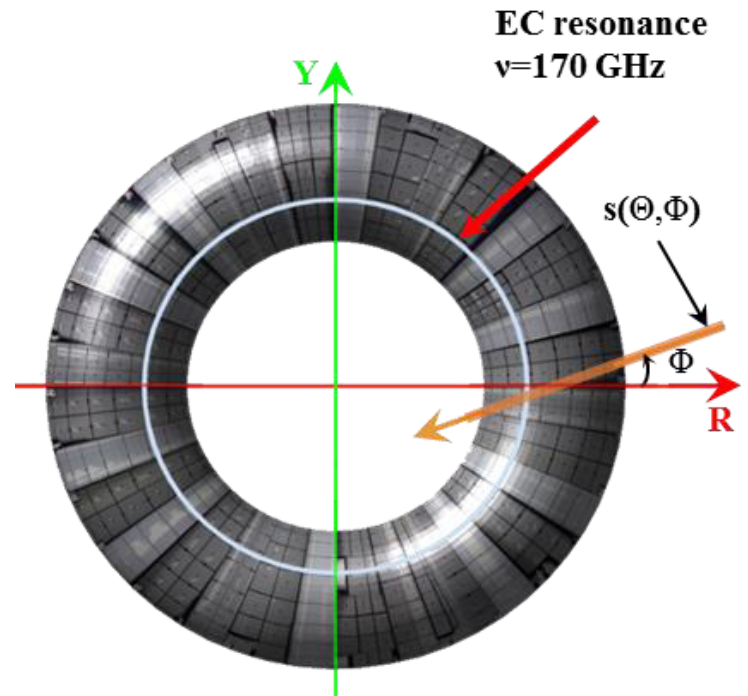

(a)

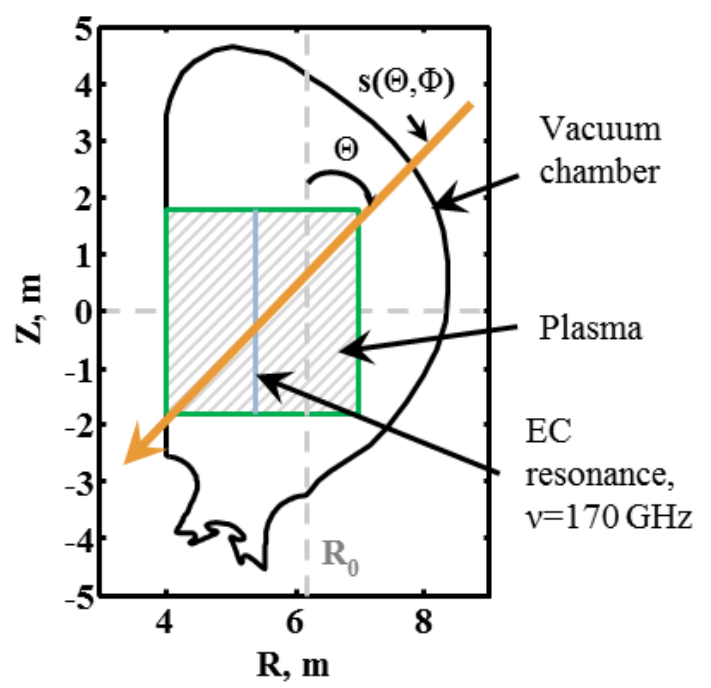

(b)

Figure 1. Electron cyclotron heating in the geometry of tokamak-reactor ITER: (a) - top view in the plane R, Y; (b) - cross-section of the tokamak in the plane $\mathrm{R}, \mathrm{Z}$. The direction of propagation of EC beam is given by the unit vector $\mathrm{s}(\Theta, \Phi)$, which depends on the angle $\Theta=\measuredangle\left(\mathbf{e}_{\mathrm{z}}, \mathbf{s}\right)$ and the toroidal injection angle $\Phi=\measuredangle\left(\mathbf{e}_{\mathrm{R}}, \mathbf{s}\right)$. Basic parameters of ITER: major torus radius $\mathrm{R}_{0}=6.2 \mathrm{~m}$, minor radius (in the equatorial plane) $\mathrm{a}=2 \mathrm{~m}$, toroidal magnetic field on the toroidal axis $\mathrm{B}\left(\mathrm{R}_{0}\right) \equiv \mathrm{B}_{0}=5.3 \mathrm{~T}$. The ECRH\&ECCD system in ITER [3]: frequency of gyrotron $v_{\text {inject }}=170 \mathrm{GHz}$, Omode, the injected power of EC waves $\mathrm{P}_{\text {inject }}=24 \mathrm{MW}$, toroidal injection angle $\Phi=20^{\circ}$.

\section{Single-pass absorption of EC radiation}

The absorption of the injected EC radiation power, $\mathrm{P}_{\text {inject }}$, can be calculated by the formula:

$$
P_{\text {absorp }}=P_{\text {inject }}\left(1-f_{O} e^{-\tau_{0, \text { eff }}}-f_{X} e^{-\tau_{X, e f f}}\right) \text {, }
$$

where $f_{O, X}$ coefficient determines the fractions of the radiation of the EC wave modes, respectively: ordinary (O-mode) and extraordinary (X-mode); $\tau_{(\mathrm{O}, \mathrm{X}) \text {,eff }}-$ effective optical thickness of the plasma column. The efficiency of the ECH absorption is defined as follows:

$$
\gamma_{\mathrm{ECH}}=\frac{P_{\text {absorp }}}{P_{\text {inject }}} .
$$

The authors of the 1D simulations [12] of plasma start-up in ITER geometry (see fig. 1) with the OGRAY code [7] for ECH calculations propose the following scaling formula for the single-pass $\mathrm{ECH}$ absorption for $10 \leq \mathrm{T}_{\mathrm{e}}[\mathrm{eV}] \leq 1000,10^{17} \leq \mathrm{n}_{\mathrm{e}}\left[\mathrm{m}^{-3}\right] \leq 10^{19}$ :

$$
\gamma_{\mathrm{ECH}}^{\mathrm{OGRAY}}=\frac{\mathrm{T}_{\mathrm{e}}[\mathrm{eV}] \cdot \mathrm{n}_{\mathrm{e}}\left[10^{19} \mathrm{~m}^{-3}\right]}{400} .
$$

It is worth noting that in OGRAY calculations [12] the distortion of electron velocity distribution function by a strong EC wave absorption was not taken into account.

A rough estimation of $\gamma_{\mathrm{ECH}}$ can be obtained from equation (1) using the analytical formulas for the effective optical thickness of the plasma column [13] (table XII, p. 1206). In the vacuum limit for the case of the O-mode propagation perpendicular to magnetic field we obtain:

$$
\gamma_{\mathrm{ECH}}^{\text {Single }}=\frac{\mathrm{T}_{\mathrm{e}}[\mathrm{eV}] \cdot \mathrm{n}_{\mathrm{e}}\left[10^{19} \mathrm{~m}^{-3}\right]}{550}
$$

The comparison of single-pass models for ECH absorption (3) and (4) is given in Fig. 2. Figure 2 shows that for plasma parameters at initial stage of discharge in ITER, $\mathrm{n}_{\mathrm{e}} \leq 0.1 \cdot 10^{19} \mathrm{~m}^{-3}$ and $\mathrm{T}_{\mathrm{e}} \leq 80 \mathrm{eV}$, the efficiency of ECH in ITER (O-mode) will be less than 1-5\%.

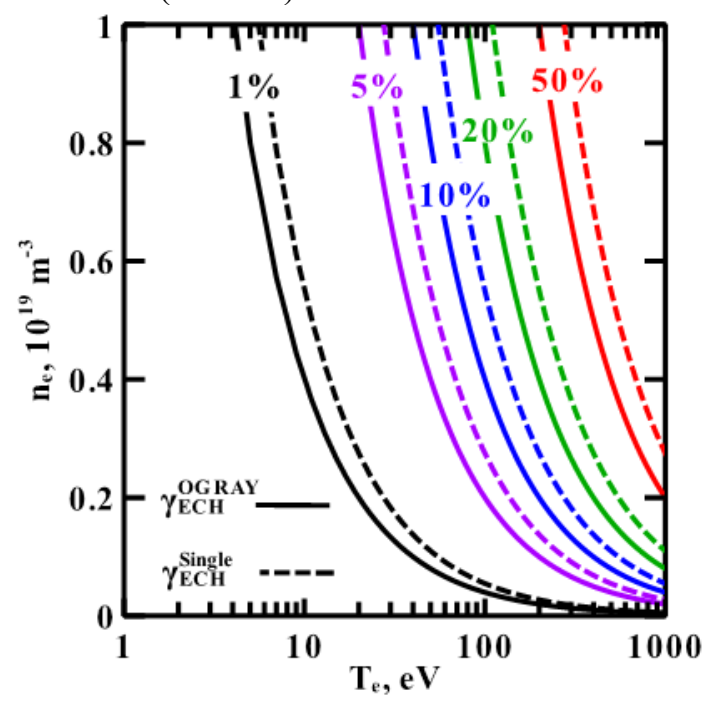

Figure 2. Comparison of the level lines of efficiency of the single-pass ECH absorption in ITER: OGRAY scaling (solid) vs eq. (4) (dashed).

Formula (4) underestimates the absorption efficiency of EC-heating by $\sim 30 \%$ in comparison with scaling (3). 


\section{A model for multi-pass absorption of EC radiation}

If the single-pass ECH absorption is small we have to consider the multi-pass absorption. We propose the following model for the multi-pass ECH absorption:

- multiple reflection of the EC wave from the wall of the vacuum chamber,

- isotropy/uniformity of the injected EC radiation intensity in plasma,

- EC mode mixing in wall reflections,

- full single-pass X-mode absorption.

The model modifies the approach of the CYTRAN code [14] and the CYNEQ code [15], [16], developed for the plasma-produced EC radiation transport at moderate and high EC harmonics and verified in the benchmarkings [17]-[19].

We consider two terms in the total absorbed power of the injected ECH: single-pass absorption of the injected EC wave (O-mode) and the above model for multi-pass absorption after first reflection of the EC wave from the wall:

$$
\mathrm{P}_{\text {absorp }}=\mathrm{P}_{\text {absorp }}^{\text {Single }}+\mathrm{P}_{\text {absorp }}^{\text {Multi }}
$$

For the case of the injected O-mode the single-pass absorption is calculated by the formula (1) with $\mathrm{f}_{\mathrm{X}}=0$ :

$$
\mathrm{P}_{\text {absorp }}^{\text {Single }}=\mathrm{P}_{\text {inject }}^{\mathrm{O}}\left(1-\exp \left[-\tau_{\mathrm{O}}^{(\mathrm{kin})}\left(\mathrm{P}_{\text {inject }}^{\mathrm{O}}\right)\right]\right) \text {. }
$$

We will use the OGRAY scaling formula (3) for the single-pass absorption, so the effective optical thickness in (6) is given by the formula:

$$
\tau_{\mathrm{O} 1}^{(\mathrm{kin})}\left(\mathrm{n}_{\mathrm{e}}, \mathrm{T}_{\mathrm{e}}\right)=-\ln \left(1-\frac{\mathrm{T}_{\mathrm{e}}[\mathrm{eV}] \mathrm{n}_{\mathrm{e}}\left[10^{19} \mathrm{~m}^{-3}\right]}{400}\right) .
$$

The multi-pass absorption can be calculated as follows:

$$
\mathrm{P}_{\text {absorp }}^{\text {Multi }}=\sum_{\varsigma=X, O} \int \mathrm{dV} \int \mathrm{d} \omega \int \mathrm{d} \boldsymbol{\Omega} \mathrm{I}_{\varsigma}(\mathbf{r}, \mathbf{n}, \omega) \cdot \boldsymbol{\kappa}_{\varsigma}(\mathbf{r}, \mathbf{n}, \omega),
$$

where $\mathrm{I}$ is the intensity of EC radiation, $\Phi=\{\omega, \mathbf{n}, \varsigma\}, \omega$ and $\mathbf{n}=\mathbf{k} / \mathbf{k}$ - wave frequency and direction of propagation, $\mathbf{k}$ - wave vector, index $\varsigma=\mathrm{X}, \mathrm{O}$ denotes, respectively, the extraordinary $(\mathrm{X})$ and ordinary $(\mathrm{O})$ wave types, $\kappa(\mathbf{r}, \phi)$ is the EC radiation absorption coefficient.

For the intensity of the EC radiation we will use semianalytical solution of the radiative transfer problem for the case of multiple reflection of radiation from the wall [14], [15], [16]. In frame of this approach we assume the isotropy/uniformity of the injected EC radiation intensity, so the eq. (8) may be rewritten in the form:

$$
\begin{gathered}
P_{\text {absorp }}^{\text {Mult }}=\sum_{\varsigma=X, O} 4 \pi \int \mathrm{dVd} \omega \mathrm{I}_{\varsigma}(\omega)\left\langle\kappa_{\varsigma}(\mathbf{r}, \mathbf{n}, \omega)\right\rangle_{\Omega}, \\
\left\langle\kappa_{\varsigma}(\mathbf{r}, \mathbf{n}, \omega)\right\rangle=\frac{1}{4 \pi} \int \mathrm{d} \boldsymbol{\Omega} \kappa_{\varsigma}(\mathbf{r}, \mathbf{n}, \omega) .
\end{gathered}
$$

From energy balance equation one can obtain (see fig. 3):

$$
\mathrm{I}_{\mathrm{X}}=\frac{\mathrm{C}_{\mathrm{O}} 4 \pi \int \mathrm{dV}\left\langle\mathrm{q}_{\mathrm{X}}\right\rangle_{\Omega}+\pi \mathrm{S}_{\text {tot }} \mathrm{R}_{\mathrm{OX}} 4 \pi \int \mathrm{dV}\left\langle\mathrm{q}_{\mathrm{O}}\right\rangle_{\Omega}}{\mathrm{C}_{\mathrm{X}} \mathrm{C}_{\mathrm{O}}-\left(\pi \mathrm{S}_{\mathrm{tot}}\right)^{2} \mathrm{R}_{\mathrm{XO}} \mathrm{R}_{\mathrm{OX}}},
$$

$$
\begin{aligned}
& \mathrm{I}_{\mathrm{O}}=\frac{\mathrm{C}_{\mathrm{X}} 4 \pi \int \mathrm{dV}\left\langle\mathrm{q}_{\mathrm{O}}\right\rangle_{\Omega}+\pi \mathrm{S}_{\mathrm{tot}} \mathrm{R}_{\mathrm{XO}} 4 \pi \int \mathrm{dV}\left\langle\mathrm{q}_{\mathrm{X}}\right\rangle_{\Omega}}{\mathrm{C}_{\mathrm{X}} \mathrm{C}_{\mathrm{O}}-\left(\pi \mathrm{S}_{\mathrm{tot}}\right)^{2} \mathrm{R}_{\mathrm{XO}} \mathrm{R}_{\mathrm{OX}}}, \\
& \mathrm{C}_{\varsigma}=4 \pi \int \mathrm{dV} \kappa_{\varsigma}+\pi\left(1-\mathrm{R}_{\varsigma \varsigma}\right), \varsigma=\mathrm{X}, \mathrm{O},
\end{aligned}
$$

where $\mathrm{q}_{\mathrm{X}, \mathrm{O}}$ is power density of ECR source, $\mathrm{S}_{\text {tot }}-$ area of vacuum chamber inner surface, $R_{\varsigma \varsigma^{\prime}}$ is the wall reflection coefficient for the incident $\varsigma$ mode and the reflected $\varsigma$ ' mode, cf. fig. 3 (we assume $\mathrm{R}_{\varsigma \varsigma^{\prime}}(\omega)=$ const).

We consider the case when the X-mode appeared in wall reflections is full absorbed in a single pass in plasma. In this case eqs. (5)-(7), (9)-(13) give the following formula for the efficiency of the ECH absorption:

$$
\begin{gathered}
\gamma_{\mathrm{ECH}}=1-\mathrm{M} \cdot \exp \left[-\tau_{\mathrm{O}}^{(\mathrm{kin})}\right], \\
\mathrm{M}=\left(1-\mathrm{R}_{\mathrm{OO}}-\mathrm{R}_{\mathrm{OX}}\right) . \\
\frac{4 \pi \int \mathrm{dV}\left\langle\kappa_{\mathrm{O}}\right\rangle_{\Omega}+\pi \mathrm{S}_{\mathrm{tot}}}{4 \pi \int \mathrm{dV}\left\langle\kappa_{\mathrm{O}}\right\rangle_{\Omega}+\left(1-\mathrm{R}_{\mathrm{OO}}\right) \pi \mathrm{S}_{\mathrm{tot}}} .
\end{gathered}
$$

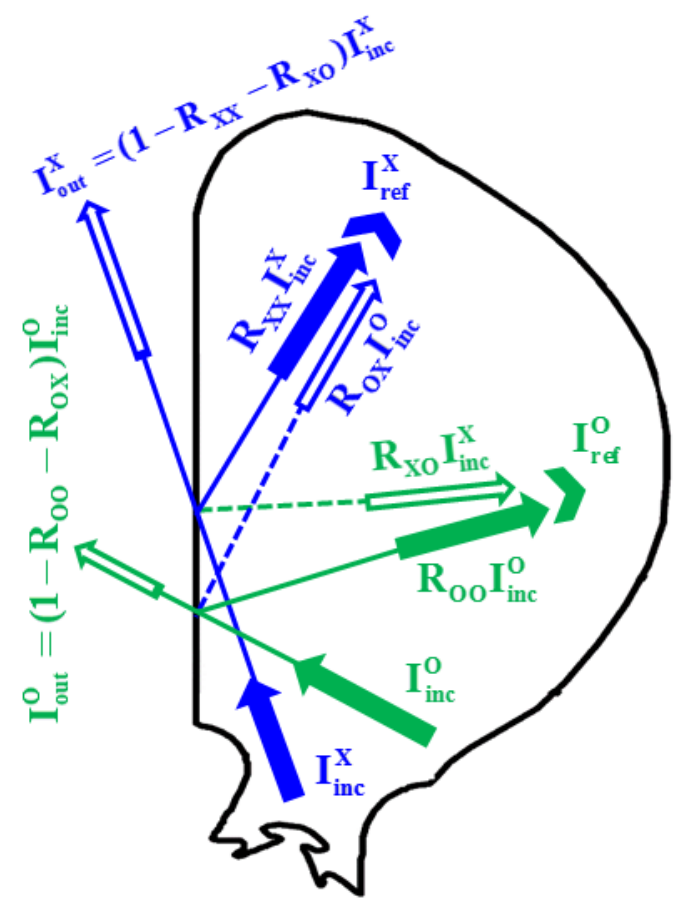

Figure 3. Schematic diagram of the boundary conditions for intensity of the EC radiation, I, for the case of mode-dependent reflection \& polarization scrambling. The subscripts 'inc' (incoming), 'ref' (reflected) and 'out' (outgoing), respectively.

The comparison of single-pass and multi-pass models for the ECH absorption efficiency is given in figs. 4-5. The dependence of the ECH efficiency on the reflection coefficients is shown in figs. 6-8. The calculations for the multi-pass model are given for the plasma shape with parameters $L_{z} \approx 3.6 \mathrm{~m}, L_{\mathrm{X}} \approx 3 \mathrm{~m}$ (see fig. 1), obtained in self-consistent calculations of the ITER start-up scenario in [12]. The absorption of the O1-mode is calculated with formulas from [13] (table XI, p. 1202).

Figure 4 illustrates the effect of increasing efficiency of absorption of the EC waves, when we take into account the multi-pass EC wave absorption (assuming complete single-pass absorption of the X-mode that appears in wall reflections). It turns out that in multi-pass 
absorption model at a fixed electron temperature the given value of ECH efficiency is achieved for lower values of electron density. Figure 5 shows the effect of an increase of ECH efficiency with increasing the fraction of the $\mathrm{EC}$ radiation converted from $\mathrm{O}$-wave to $\mathrm{X}$-wave in wall reflections. It is important to study the dependence of the EC heating efficiency simultaneously on two parameters - wave reflection coefficient from the wall and the proportion of radiation passing from $\mathrm{O}$ - to $\mathrm{X}$ mode in wall reflections.

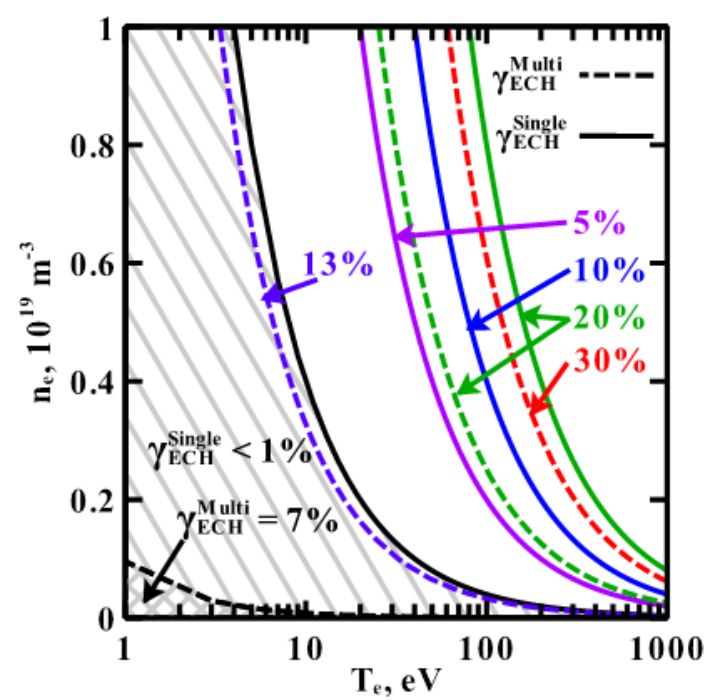

Figure 4. Comparison of the level lines of the ECH power absorption efficiency, $\gamma_{\mathrm{ECH}}$ : single-pass O-wave absorption scaling, obtained in [12] with OGRAY code (solid); multi-pass absorption model for the coefficient of O-wave reflection from the wall $\mathrm{R}_{\mathrm{OO}}=0.6$ and coefficient of $\mathrm{O}$-mode to $\mathrm{X}$-mode conversion in wall reflections $\mathrm{R}_{\mathrm{OX}}=0.1$ (dashed).

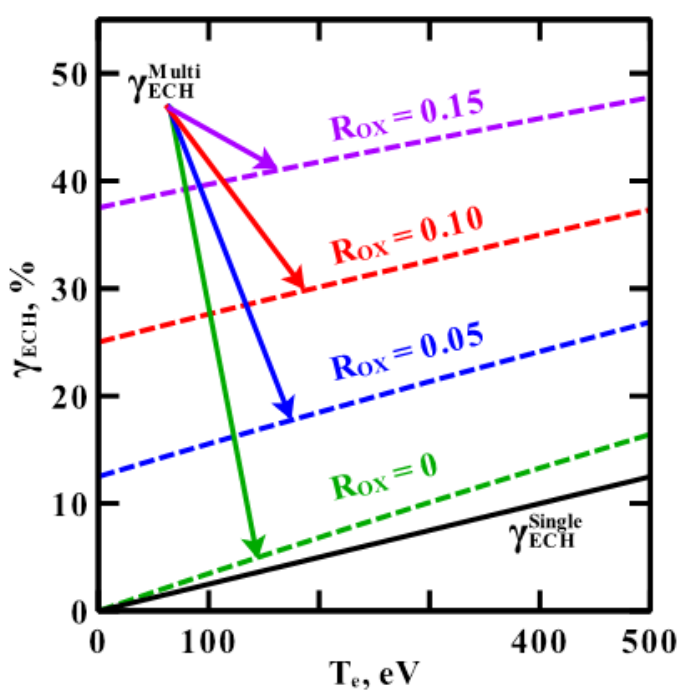

Figure 5. Comparison of the efficiency of the single-pass ECH absorption model (OGRAY scaling, solid) and the suggested multi-pass absorption model (dashed) as a function of electron temperature for $\mathrm{n}_{\mathrm{e}}=0.110^{19} \mathrm{~m}^{-3}$. Calculations in the multi-pass absorption model are carried out for $\mathrm{R}_{\mathrm{OO}}=0.6$ and several values of $\mathrm{R}_{\mathrm{OX}}$.

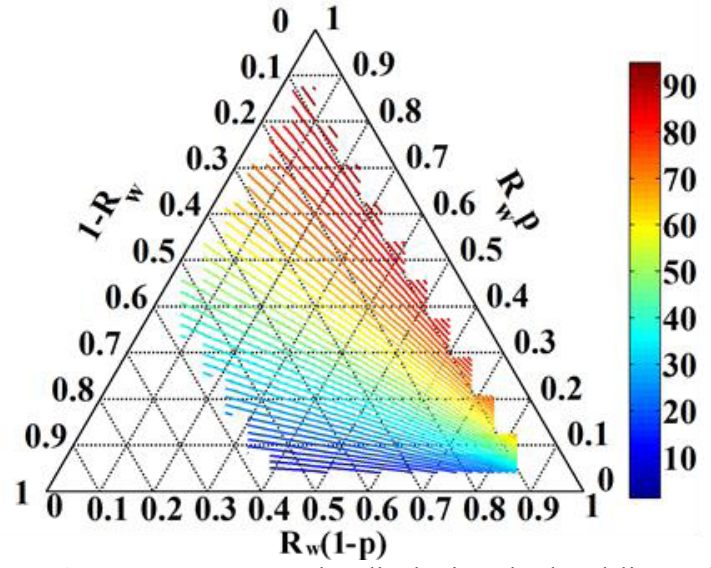

Figure 6. Ternary contour plot displaying the level lines of the efficiency of ECH absorption in ITER in the multi-pass absorption model for $\mathrm{T}_{\mathrm{e}}=10 \mathrm{eV}, \mathrm{n}_{\mathrm{e}}=0.210^{19} \mathrm{~m}^{-3}$. Parameter $\mathrm{p}$ is the polarization scrambling parameter (percentage of radiation converted from one mode to another in the wall reflection).

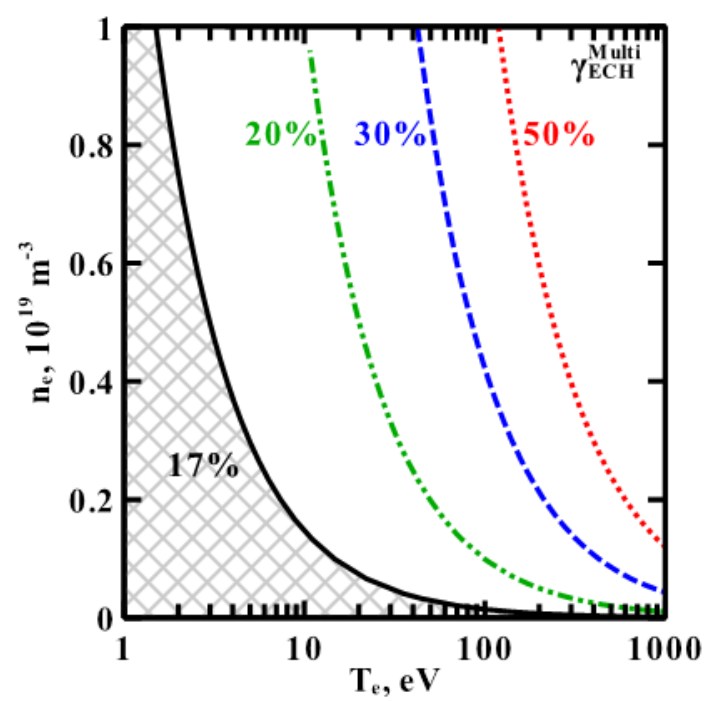

Figure 7. The level lines of the ECH power absorption efficiency, $\gamma_{\mathrm{ECH}}$, in the multi-pass absorption model for $\mathrm{R}_{\mathrm{OO}}=0.7, \mathrm{R}_{\mathrm{OX}}=0.05$.

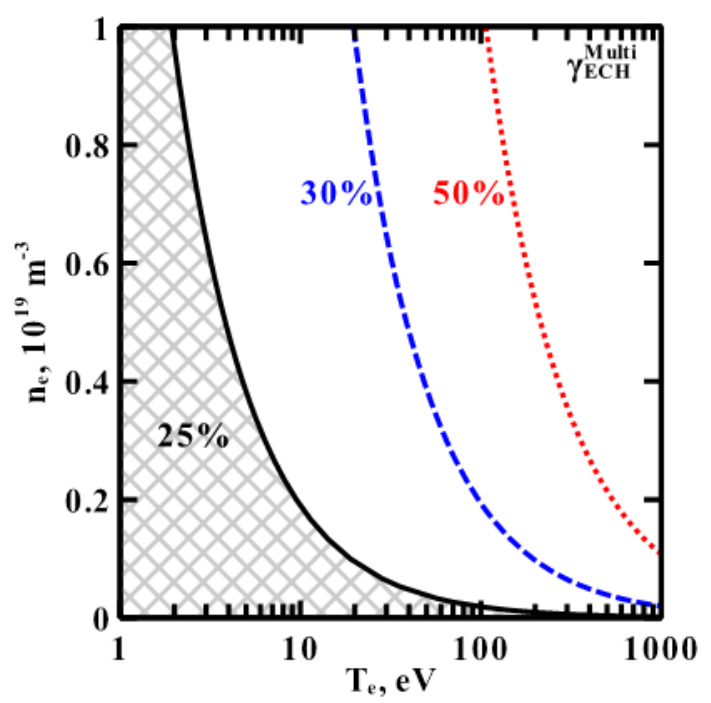

Figure 8. The level lines of the $\mathrm{ECH}$ power absorption efficiency, $\gamma_{\mathrm{ECH}}$, in the multi-pass absorption model for $\mathrm{R}_{\mathrm{OO}}=0.6, \mathrm{R}_{\mathrm{OX}}=0.1$. 
In the case of small absorption of the O-mode, eqs. (14)(15) are simplified to give as follows (see fig. 9):

$$
\gamma_{\mathrm{ECH}}^{\text {Multi }}=\frac{\mathrm{R}_{\mathrm{OX}}}{1-\mathrm{R}_{\mathrm{OO}}} .
$$

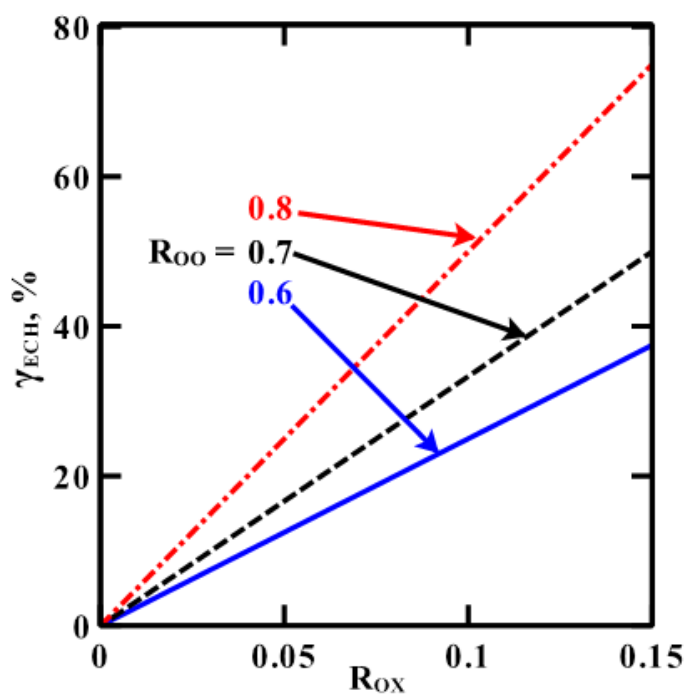

Figure 9. The efficiency of ECH absorption in ITER in the multi-pass absorption model in the case of a weak absorption of the O-wave.

\section{Conclusions}

A model for calculating the efficiency of the multi-pass absorption of the EC heating power in tokamaks at initial stage of discharge is proposed. The single-pass absorption of injected EC radiation is evaluated with the scaling [12] obtained using the OGRAY code. For the subsequent multi-pass absorption, after first reflection of the EC wave from the wall of the vacuum chamber, we develop a model based on the assumption of the isotropy/uniformity of the respective $\mathrm{EC}$ radiation intensity in plasma (a semi-analytical solution of the radiative transfer problem for the case of multiple reflection of radiation from the wall). The model modifies the approach of the CYNEQ code [15], [18] developed for the plasma-produced EC radiation transport at high EC harmonics and verified in the benchmarkings [17]-[19]. In the frame of this approach, we consider the following case: (a) multiple reflection of injected EC wave (O-mode) from the wall; (b) polarization scrambling in wall reflections; (c) full single-pass absorption of the X-mode. Our parametric analysis of the efficiency of multi-pass absorption of injected EC radiation for typical values of the electron temperature and density at the initial stage of discharge in ITER-like tokamak shows strong dependence on the $\mathrm{O}-\mathrm{X}$ conversion in wall reflections.

The performed parametric analysis of the efficiency of ECH absorption in ITER at initial stage of discharge indicates that further investigation of the role of the multi-pass absorption effects is needed. The considered case of the full single-pass $\mathrm{X}$-mode conversion is an estimation of the most optimistic scenario. Furthermore, the effects of multi-pass absorption of injected EC radiation were studied within the transport model which allows only for the EC waves.

Acknowledgements. The authors are grateful to A.V. Timofeev, A.V. Zvonkov for helpful discussions, A.Y. Kuyanov, for providing with the OGRAY code for the use in the frame of the DINA suite of codes.

The work is supported by the State Corporation ROSATOM and the Council of the Russian Federation Presidential Grants for State Support of Leading Scientific Schools (grant no. NSh-3328.2014.2).

\section{References}

1. B. Lloyd, P.G. Carolan, C.D. Warrick, Plasma Phys. Controlled Fusion 38, 1627-1643 (1996)

2. ITER Physics Expert Group on Energetic Particles, Heating and Current Drive, ITER Physics Basis Editors, Nuclear Fusion 39, 2495 (1999)

3. T. Omori, M.A. Henderson, F. Albajar, S. Alberti, et al., Fusion Engineering and Design 86, 951-954 (2011)

4. J. Stober, G.L. Jackson, E. Ascasibar, Y.S. Bae, et al., Nuclear Fusion 51, 083031 (2011)

5. R. Prater, Physics of Plasmas 11, 2349-2376 (2004)

6. H.P. Laqua, W7-as Team, ECRH-Group, AIP Conference Proceedings 694, 15-23 (2003)

7. A.V. Zvonkov, A.Y. Kuyanov, A.A. Skovoroda, A.V. Timofeev, Plasma Physics Reports 5, 389-400 (1998)

8. A.P. Smirnov, R.W. Harvey, K. Kupfer, Bulletin of the American Physical Society 39, 1626 (1994)

9. R.W. Harvey, M.G. McCoy, IAEA Technical Committee Meeting on Advances in Simulation and Modeling of Thermonuclear Plasmas 489-526 (IAEA Institute of Physics Publishing, 1992)

10. Y.S. Bae, A.C. England, Journal of the Korean Physical Society 51, 1313-1319 (2007)

11. K. Hada, Japan-Korea Workshop on Physics and Technology of Heating and Current Drive (2013)

12. R.R. Khayrutdinov, A.Y. Kuyanov, V.E. Lukash, A.V. Zvonkov, $38^{\text {th }}$ EPS Conference on Plasma Physics 35G, P2.085 (ECA, 2011)

13. M. Bornatici, R. Cano, O. De Barbieri, F. Engelmann, Nuclear Fusion 23, 1153 (1983)

14. S. Tamor, Science Applications, Inc. Report SAI023-81-189LJ/LAPS-72 (La Jolla, CA, 1981)

15. A.B. Kukushkin, $14^{\text {th }}$ IAEA Conference on Plasma Physics and Controlled Nuclear Fusion Research 2, 35-45 (IAEA, 1992)

16. A.B. Kukushkin, P.V. Minashin, A.R. Polevoi, $23^{\text {rd }}$ IAEA Fusion Energy Conference ITR/P1-34 (2010)

17. F. Albajar, M. Bornatici, F. Engelmann, A.B. Kukushkin, Fusion Science and Technology $\underline{\mathbf{5 5}}$ 76-83 (2009)

18. A.B. Kukushkin, P.V. Minashin, $24^{\text {th }}$ IAEA Fusion Energy Conference TH/P6-25 (2012)

19. A.B. Kukushkin, P.V. Minashin, A.R. Polevoi,

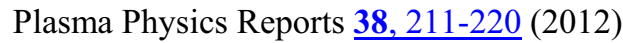

